\title{
Towards the Optimal RF Sensing Strategy for IoT Applications: An Ice Sensing Case Study
}

\author{
Mahmoud Wagih, Member, IEEE, and Junjie Shi
}

\begin{abstract}
Remote ice detection has recently emerged as an application of Radio Frequency (RF) sensors. While RF sensing is a feasible approach used for detecting various stimuli, the optimal system architecture and design strategy for RF-based sensing in future Internet of Things (IoT) systems remains unclear. In this paper, we propose a systematic methodology for designing an RF-based sensing system, applicable to a plethora of IoT applications. The proposed methodology is used to design printable antennas as highly-sensitive sensors for detecting and measuring the thickness of ice, demonstrating best-in-class sensory response. Antenna design is investigated systematically for wireless interrogation in the $2.4 \mathrm{GHz}$ band, to support a variety of IoT protocols. Following the proposed methodology, the antenna's realized gain was identified as the optimum parameterunder-test. The developed loop antenna sensor exhibits a high linearity, resilience to interference, and applicability to different real-world deployment environments, demonstrated through over $90 \%$ average ice thickness measurement accuracy and at least $5 \mathrm{~dB}$ real-time sensitivity to ice deposition.
\end{abstract}

Index Terms-Antennas, Antenna Gain, Antenna Sensors, Ice Sensing, Impedance Matching, RF ice sensing, RFID, Materials, Relative Permittivity Measurement, Wireless Sensing

\section{INTRODUCTION}

$\mathbf{R}$ EMOTELY monitoring the accumulation of ice and frost is of great importance to smart cities [1], industrial [2], and environmental sensing [3] applications. For example, in renewable wind power farms, ice formation on wind turbines can reduce their power output by up to $30 \%$ over a year, in a region prone to icing events [4]. In the United States, around 250,000 house-holds are affected by frozen water pipes annually [5]. Furthermore, In remote and environmental sensing, measuring the thickness of ice sheets can help monitor climate change [6], and can be used to predict avalanches [7].

Radio Frequency (RF) sensing has attracted significant research interest [8], building upon well-established technologies such as RFID [9], and microwave resonators [10]. Vital life signals [11], gases [12], [13], structural health [14], compression [8], temperature [15], and food quality [16] have all been monitored using RF-based sensing for an "RF-enabled" sensory Internet of Things (IoT). Various topologies and sensing devices from resonant waveguiding structures [10], to radiating antennas have been used for RF sensing [13],

This work was supported by the UK Engineering and Physical Sciences Research Council (EPSRC) under Grant EP/P010164/1. (Corresponding author: Mahmoud Wagih)

The authors are with the School of Electronics and Computer science, University of Southampton, Southampton, SO17 1BJ, U.K. (email:mahm1m19@soton.ac.uk)

Digital Object Identifier: , datasets used in this paper will be made available from the University of Southampton repository at DOI: X
[17]. Nevertheless, there is no standardized methodology for designing RF sensors, or a detailed comparison on the effect of different "variables" on the system's performance. These variables and design decisions include the choice of frequency, the sensing element design, or the RF parameters, such as scattering (s)-parameters or antenna radiation properties, to be interrogated by the read-out circuit. This ambiguity results in limited adoption of RF-based sensing in real IoT systems. To illustrate, in certain designs, the resonant sensors may act as radiators (i.e. antennas) [18], which may restrict their real-world deployment due to frequency spectrum regulations requiring sensor re-design. As multiple research efforts have been carried out to present holistic and standardized approaches to IoT sensing [19], a similar study focusing on standardizing the design of RF sensors is required.

Ice detection and remote thickness measurements represents an interesting case-study for RF-based sensors [20], [21]; the optical translucency of ice and its low permittivity imply that highly sensitive approaches beyond manual inspection are required. Near infrared (NIR) cameras [22] and fibre optic sensors have been proposed for detecting the formation of ice [23], yet, they cover a small area and are generally less sensitive than microwave sensors [21]. Microwave twoport resonators were proposed as highly-accurate sensors for ice [21], [24], surpassing other visual and optical methods. As the aforementioned approaches require active sampling circuitry, flexible passive RFID tags were investigated for accurate wireless ice detection [20]. We then proposed a 2.4 GHz wire-type antenna as an ice sensor, observing a correlation between the antenna's gain and ice thickness [25]; aperture-type patch antenna sensors were also proposed based on gain measurements at $2.4 \mathrm{GHz}$ [26], and based on $S_{11}$ measurements at $4 \mathrm{GHz}$ [27]. Nevertheless, as with most RF sensing applications, the effects of choosing an RFID antenna [20], a $50 \Omega$ antenna [25]-[27], or a two-port resonator [21], [24], on the sensor's performance remains unclear, due to the discussed ambiguity in RF sensing mechanisms, and the lack of a clear standardized approach to identify the optimal sensing parameter. Furthermore, while both the antenna's gain and impedance were used for sensing in [26] and [27], respectively, it is unclear which RF parameter yields the highest accuracy.

In this paper, we propose a standard methodology for designing an RF-based sesnor for future IoT applications, and apply it to ice thickness measurements resulting in the highest accuracy and sensitivity compared to previous work. The key contributions of this paper can be summarized as:

1) A standard methodology for designing RF sensors for different applications, circuits, and frequency bands; 
2) A detailed investigation of the influence of antenna/RF sensor parameters on a system's functionality, susceptibility to interference, and complexity;

3) A high sensitivity flexible antenna for ice detection and remote thickness measurements, comparing favorably with state-of-the-art. implementation

Section II introduces the proposed flow for designing RF sensors. In Section III, different antennas are simulated, comparing their performance as ice sensors and evaluating the suitability of different antenna parameters for sensing applications. The fabricated sensors are then characterized experimentally in Section IV, where the overall design procedure is evaluated and discussed in the context of an RF-enabled sensing IoT, and compared to similar systems in literature.

\section{RF-Sensing Methodology}

Antenna-based sensing, and more generally RF-based sensing, relies on a change in the dielectric or conductive properties of a material to change the performance of the RF device [10]. This change is typically observed through the s-parameters matrix [10], [18], or through the far-field gain [20], of a sensing antenna. To explain, the real-permittivity $\left(\epsilon_{r}\right)$ results in a resonance shift due to the increase in the capacitive element of the antenna/resonator's equivalent circuit. Similarly, a change in the $\tan \delta$ of the material results in a change in the antenna/resonator's gain or quality (Q)-factor. Both changes, to $f_{r}$ and $|G|$ or $\mathrm{Q}$, can be used to detect changes in the environment.

The most common of RF sensors are two-port resonators, demonstrating high sensitivity for microfluidic and biomedical applications [10], and previously used for detecting freeze events [21]. Such sensor a could be interrogated using a high-sensitivity readout circuit as shown in Fig. 1(a); in a lab environment, this is typically achieved using a Vector Network Analyzer (VNA) or a similar broadband sampling circuit such as a software defined radio [28]. Alternatively, as resonators could be designed with a filter-like response, they could be integrated in chipless RFID tags by connecting them to broadband monopole antennas [12]. Antennas can also be used to directly detect changes in the environment through the gain [20], [26] or the $S_{11}$ response [27].

To read-out the sensory response of an RF resonator or an antenna, different approaches could be used, as shown in Fig. 1. The sensing topology and subsequently the parameterunder-test (PUT) need to be selected based on the hardware, frequency, and power restrictions, as in the proposed RF-based sensing methodology flow, shown in Fig. 2. To begin with, the frequency spectrum allocation restricts the operation of the sensor. To explain, IoT sensors are only allowed to radiate power in the industrial scientific and medical (ISM)-bands, e.g. $868 / 815 \mathrm{MHz}, 2.4$ and $5.8 \mathrm{GHz}$, and the $3-10 \mathrm{GHz}$ UWB spectrum. Therefore, unless operating in the UWB spectrum, wireless sensors can only be interrogated in narrow ISMbands. This prohibits the detection of the resonant frequency $f_{r}$, which is impossible to identify without a broadband sweep [20]. Furthermore, two-port resonators based on microstrip technology and operating outside such bands need to ensure
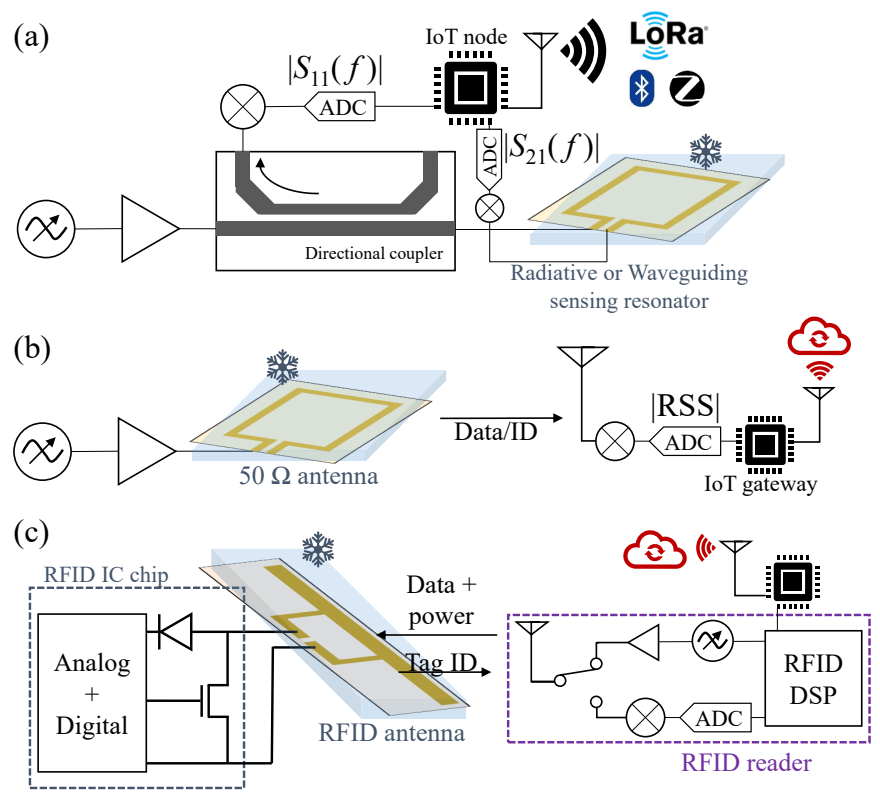

Fig. 1. RF-based sensing approaches and their integration in IoT networks: (a) one/two-port s-parameter sensing [10]; (b) wireless sensing through a $50 \Omega$ matched antenna gain (this work, [26]); (c) wireless sensing through a batteryless RFID tag's complex- $Z$ antenna gain [20].

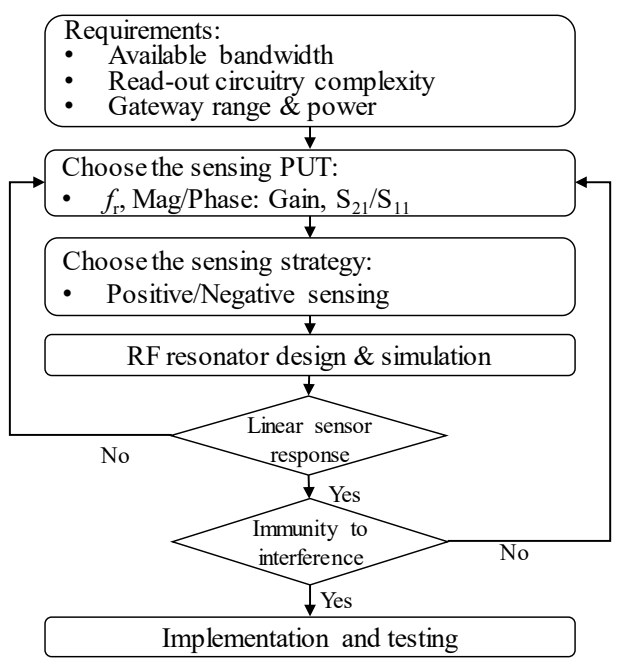

Fig. 2. RF-based sensing approaches and their integration in IoT networks: (a) one/two-port s-parameter sensing; (b) wireless sensing through a $50 \Omega$ matched antenna gain; (c) wireless sensing through a battery-less RFID tag's complex- $Z$ antenna gain.

full shielding, where microstrip lines are known to radiate with up to $-20 \mathrm{dBi}$ gain [29]. Therefore, should s-parameter-based sensing, shown in Fig 1(a), be adopted, the operation has to be restricted to an ISM-band, or utilize a fully-shielded nonradiative $\mathrm{RF}$ resonator.

The second restriction relates to the integration with practical hardware such as transmitters, power amplifiers (PAs), and detectors or demodulators, which affects the PUT . As shown in Fig. 1(a), an additional complex and area-consuming component, a directional coupler, is needed to measure the reflection coefficient, $S_{11}$, of a resonator. $S_{21}$ measurement on the other hand are simpler and only require a detector or a 


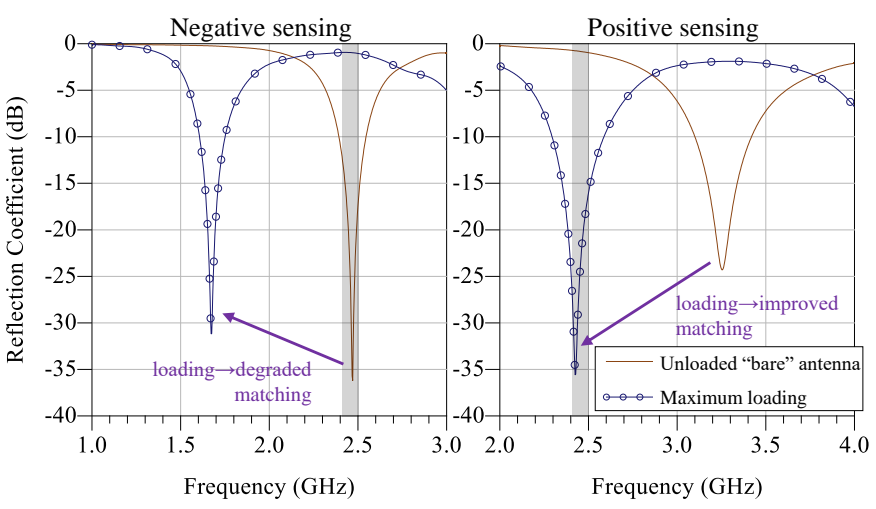

Fig. 3. The principle of positive and negative RF sensing demonstrated through the reflection coefficient $\left(S_{11}\right)$ of a resonant antenna sensor; shaded region indicates the read-out frequency.

down-converter, for measuring the amplitude of the received signal at different frequencies. In most studies, both $S_{11}$ and $S_{21}$ measurements of sensors' response are carried out using a VNA. This enables resolving differences around $0.1 \mathrm{~dB}$ [26], which could translate to under $5 \mathrm{mV}$ changes in a typical low-cost RF power detector's output, making it impractical for low-cost IoT implementations based on off-the-shelf parts. The antenna gain on the other hand could be detected wirelessly, through the relative signal strength (RSS) of an active or backscattered transmission [11], which enables a plethora of RFID-based sensing applications.

Following the identification of the sensing approach and the wireless regulations, the RF sensing element is designed, as detailed in the next section. The design process involves iterative 3D full-wave electromagnetic simulation to observe the sensor's response to the stimulant for multiple PUTs. At this stage, the performance of positive and negative sensing approaches can be investigated. To explain, a $\Re\left\{\epsilon_{r}\right\}$ will result in a frequency shift in the resonant response of an antenna or a two-port sensor. The antenna could be designed to achieve the maximum gain and minimum $S_{11}$, under loading, which can improve the read-range of a passive sensor [20], i.e. "positive sensing". Alternatively, the antenna sensor could be designed to achieve the best gain and $S_{11}$ prior to loading, where the stimulus is detected through "detuning", acting as a "negative sensor". Fig. 3 illustrates the operation of a positive and a negative resonant sensor, through the $S_{11}$. The process of observing the sensor's linear response, evaluating its susceptibility to the anticipated sources of interference, and experimental evaluation is presented in the next sections.

\section{Antenna Sensor Design and Simulations}

The proposed sensor needs to operate in a license-free band, as well as have a response which could be interrogated remotely. Following the methodology in Fig. 2, the key variables which need to be selected to realize the RF-based solution for ice sensing applications are:

1) Parameter-Under-Test (PUT): in an antenna/RF-based sensor. This choice implies the required read-out circuit and can complicate a system beyond typical low-cost IoT hardware.
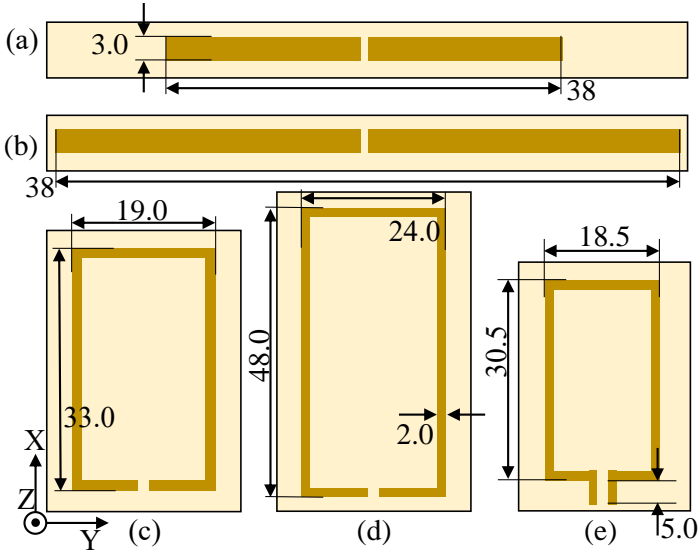

Fig. 4. Layout and dimensions of the investigated sensing antennas: (a) positive sensing dipole; (b) negative sensing dipole; (c) positive sensing loop; (d) negative sensing loop; (e) positive sensing loop with extended connector leads.

2) Antenna/resonator design: the active sensing element geometry, materials and its interaction with the loading stimulant. This can maximize and linearize the sensory response in the region of interest.

3) Positive vs. negative sensing: where the PUT either increases or decreases in response to the stimulant. This can improve the system's immunity to interference, and linearize the sensory response of the antenna's parameters in the range of interest.

\section{A. Sensitivity and Linearity Analysis}

Comparing the wire-type dipole in [20] to the aperture type patches in [27] and [26], a wire-type antenna generally exhibits higher sensitivity to ice. Two wire-type antenna designs have been simulated in CST Microwave Studio to evaluate their sensitivity and the ideal PUT, based on the sensing flow proposed in Fig. 2. The antennas are a magnetic loop, and an electric dipole. The dimensions of the antennas are shown in Fig. 4. Following on the proposed concept of positive and negative sensing, two variants of each antenna have been designed. The first variant is designed to resonate and match $50 \Omega$ at $2.4 \mathrm{GHz}$, in free space, i.e. before ice-loading. The second variant is designed to match $50 \Omega$ at $2.4 \mathrm{GHz}$ under maximum loading, this is when the antenna is completely covered in a thick layer of ice. The "thick layer" is defined as the thickness beyond which the gain and impedance response of the antenna stops varying, this was determined through the CST model, by varying the ice superstrate thickness, to be around $50 \mathrm{~mm}$-thick.

As the ice is intended to act as a superstrate which shifts the antenna's frequency response and subsequently alter the gain, an ice layer of varying thickness $t(0.1-50 \mathrm{~mm})$ has been simulation on-top of the antennas. As previously reported thickness sensors were demonstrated for sub=10 mm thickness measurements, the proposed methodology aims to significantly improve the measurement range through antenna design. The dielectric properties of ice are $\epsilon_{r}=3.2$ and $\tan \delta=0.0009$ [30]. The ice layer is $40 \times 40 \mathrm{~cm}$, substantially larger than the antenna, to mimic the effects of a full ice cover. 

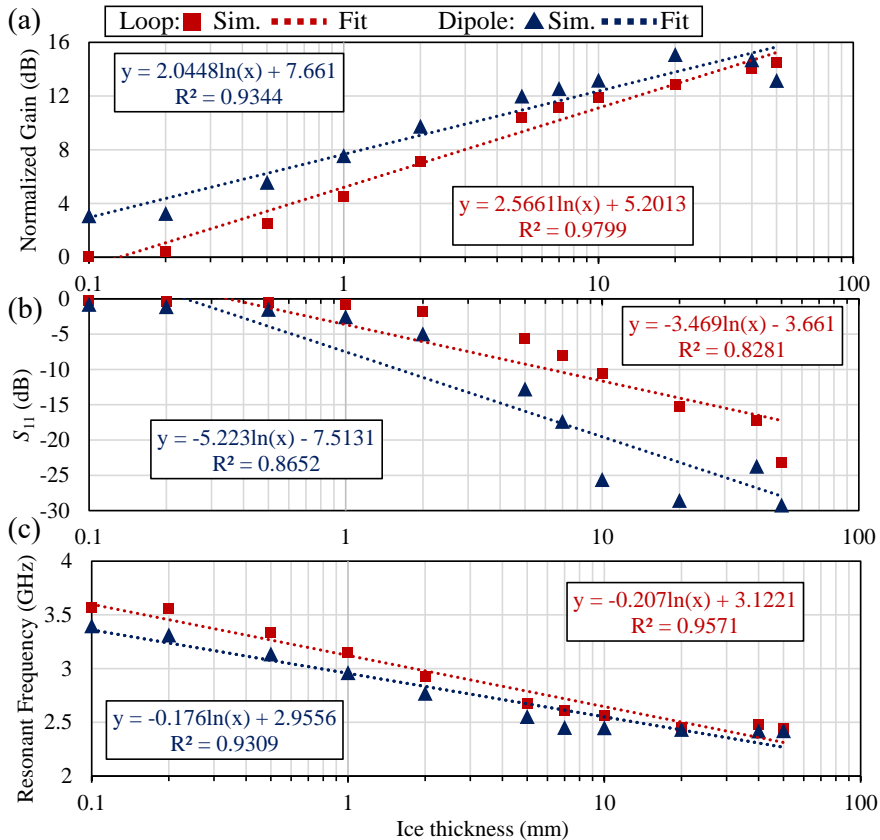

Fig. 5. Simulated antennas' PUTs positive sensing: (a) normalized gain; (b) reflection coefficient; (c) resonant frequency, showing a highly-linear response for the loop antenna for all PUTs.
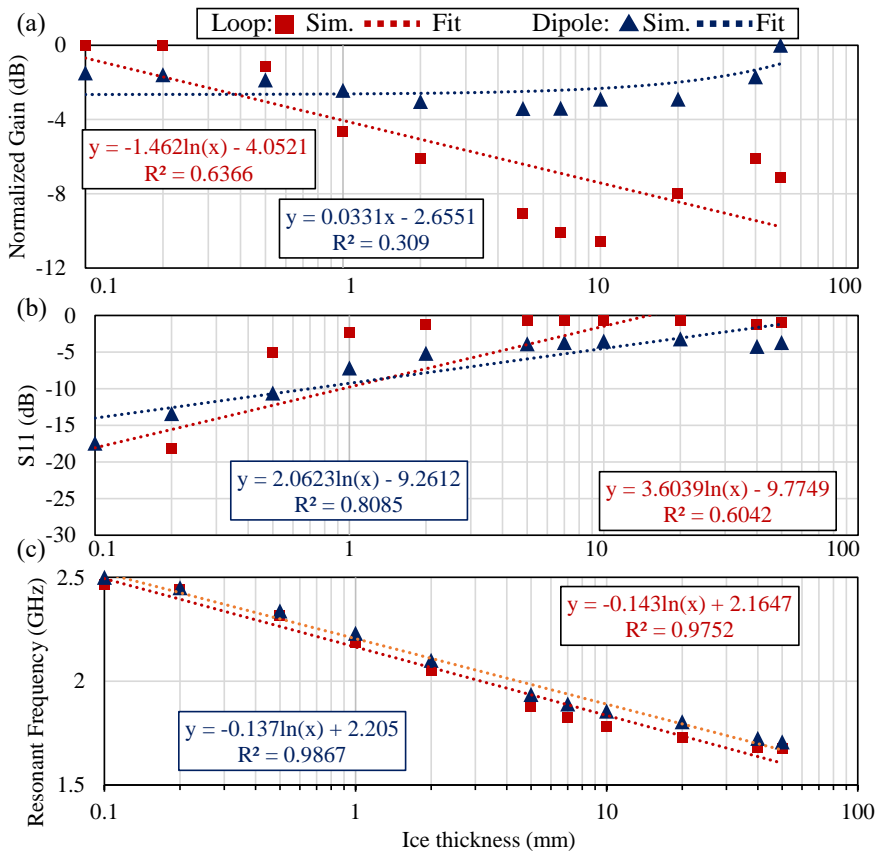

Fig. 6. Simulated antennas' PUTs for negative sensing: (a) normalized gain; (b) reflection coefficient; (c) resonant frequency, showing inferior sensory response in $G$ and $S_{11}$ compared to positive sensing.

The antenna's $S_{11}$ response and far-field radiation properties have been simualted for $0<t<50 \mathrm{~mm}$. Fig. 5 and 6 show the simulated PUTs for positive and negative sensing, respectively, for both the loop and the dipole antennas.

Observing the fitted relations in Fig. 5 and 6 , the $\mathrm{R}^{2}$ exhibits the closest fit for positive sensing, where negative sensing results in $\mathrm{R}^{2}<70 \%$ for the $\Delta G$ and $S_{11}$, unsuitable

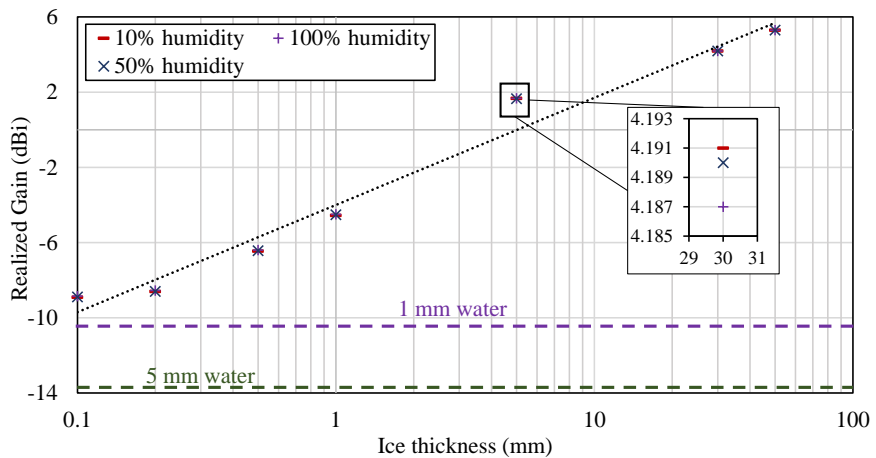

Fig. 7. Simulated gain-thickness response of the positive-sensing loop for varying humidity levels and water levels.

for thickness measurements. On the other hand, a broadband sensor which monitors $f_{r}$ is expected to maintain a high sensitivity and linearity for both positive and negative sensing. Comparing the $S_{11}$ to $G$, it can be observed that for $t>10 \mathrm{~mm}$, the $\left|S_{11}\right|$ becomes increasingly non-linear, making it less suited to estimating the thickness of thick ice layers. This is in line with the result presented in [27], where the patch's $S_{11}$ was found to only be suitable for sensing $t<7 \mathrm{~mm}$.

Given their highly linear response, the resonant frequency and the antenna gain can be chosen as an ice detection PUT. Selecting $f_{r}$ as the PUT will restrict the real-world implementation to the topology in Fig. 1(a), with a shielded resonator, unless the antenna is scaled to operate in the UWB (3-10 GHz) spectrum. On the other hand, choosing the gain as the PUT enables the sensor to be realized using either of the topologies in Fig. 1(a) and (b).

\section{B. Interference Susceptibility and Reliability}

The final step prior to prototyping, as shown in Fig. 2, is investigated the sensor's immunity to false-reads or misses. This could be due to external environmental factors, such as being loaded with water or changing ambient conditions.

The relative permittivity of the polyimide (Kapton) substrate is dependent on the humidity level, which could shift the resonance of the proposed RF ice sensing antenna [31]. Polyimide's $\epsilon_{r}$ increases linearly with humidity, where $\epsilon_{r}$ equals $3.1,3.5$, and 3.8 , for $10 \%, 50 \%$, and $100 \%$ humidity levels, respectively [32]. The aforementioned values were used to simulate the antenna's PUT, the gain, for varying ice thicknesses. In Fig. 7, it can be observed that the gain change due to humidity is insignificant compared to the ice superstrate, which validates the sensor's immunity to humidity-induced interference. As for temperature effects, the change in the permittivity of Kapton is significantly lower than that caused by humidity [32], which has a minimal influence on the gain, as shown in Fig. 7. Therefore, the printed antenna's response will not change for different temperatures.

The other source of interference expected in an ice sensor is water droplet accumulation on the surface of the sensor. Water is characterized by a very high $\epsilon_{r}$ compared to ice [30], therefore, a thin layer of water over the sensor may result in a similar frequency shift to that caused by a thick ice layer, 


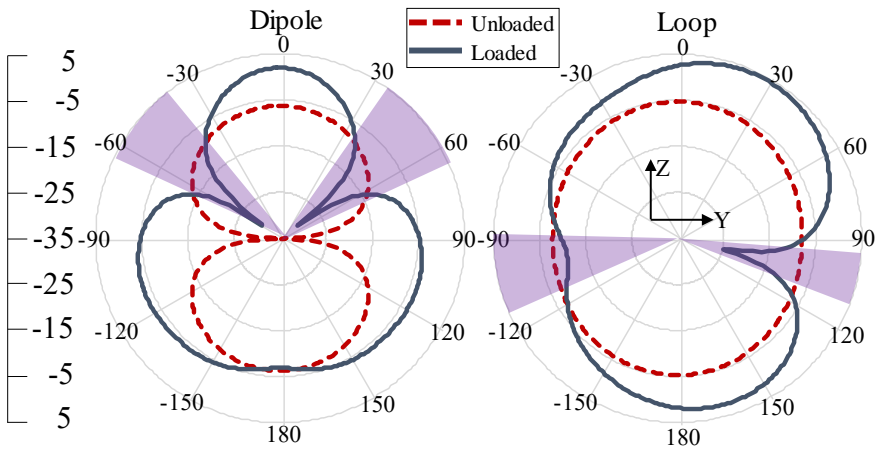

Fig. 8. Loaded (solid) and unloaded (dashed) gain patterns of the positive sensing dipole (left) and the loop (right) sensors; the shaded regions indicate the angles at which the loaded gain is lower than the unloaded gain.

causing a false match. Fig. 7 shows the simulated antenna's gain for 1 and $5 \mathrm{~mm}$ of water, where it can be observed that for both cases the antenna's gain is lower than that under all conditions of ice loading. This is attributed to the high conductivity of water which results in a low antenna efficiency due to absorption by the water molecules.

The observed response to water highlights the benefits of the proposed positive sensing methodology, as well as the chosen PUT. To explain, the presence of water on the sensor results in a matched $S_{11}<-10 \mathrm{~dB}$, which shows that should the $S_{11}$ be selected as the PUT, the sensor will not be able to distinguish frost events from water droplets caused by rain or dew. Furthermore, should a negative sensing approach be adopted, i.e. where the gain reduces in response to the ice, then the presence of water will also result in a false-match, where the presence of water will always be interpreted as a thick layer of ice, due to water's high $\tan \delta$. Therefore, according to the decision-making strategy introduced in Fig. 2, selecting the realized gain as the PUT is more suitable for remote sensing of the presence of ice than the resonant frequency.

Selecting the gain as the PUT and positive sensing as the design strategy, the final parameter which control the robustness of such a wireless antenna sensor is the radiation pattern. To explain, the angular direction of the peak gain needs to be stable for the gain change to be detected wirelessly. Otherwise, if the direction of the antenna's main lobe changes, wireless detection will not be possible and the change in the gain will not be read successfully by the receiver, resulting in angular "blind spots" in the antenna's scanning beamwidth. Fig. 8 shows the simulated realized gain pattern of the positive sensing loop and dipole, before and after $40 \mathrm{~mm}$-thick ice loading, where the blind spots are shaded.

Observing the main-lobe direction for both antennas, it can be seen that the loop's main-lobe direction is maintained for $-90<\theta<90$, indicating that the sensor's output will be consistent across the broadside hemisphere. On the other hand, the dipole's main beam changes its angular response. Therefore, should the dipole-based sensor be interrogated around $\pm 30<\theta< \pm 60$ (the shaded region), the presence of ice will not be detected due to the change in the radiation pattern. As a result, it is expected that the loop antenna will exhibit This will be experimentally validated in the next

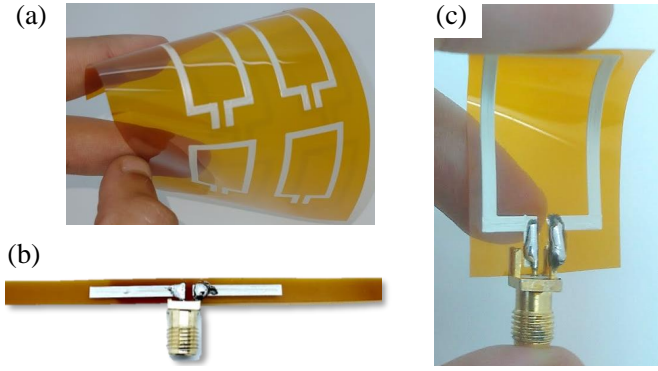

Fig. 9. Photographs of the fabricated antenna sensors: (a) printed traces on polyimide; (b) connectorized dipole antenna; (c) connectorized loop antenna.

section, by observing the sensor's response at $\theta \approx 30^{\circ}$.

\section{SEnsor Fabrication And Measurements}

\section{A. Additively-Manufactured Sensors Characterization}

The designed antennas have been fabricated on a flexible polyimide substrate of $75 \mu \mathrm{m}$ thickness. Direct-write dispenser printing, recently demonstrated for energy harvesting [33] and IoT antenna prototyping [34], has been utilized to deposit the silver tracks on the organic polymer substrate. The printer used is a commercially-available Voltera V-one printer, intended for rapid and low-cost PCB prototyping. The designed antenna traces are directly printed on the polyimide substrate, adhered to a planar surface, without the need for a screen or a photolithography mask. The printed silver traces are cured at $170^{\circ}$ for 50 minutes, using a standard hotplate. Once cured, SMA connectors are mounted using low-temperature solder and encapsulated using Kapton tape which enables a reliable connection to other coaxial components such as test cables Fig. 9(b) and (c) show the connectorized dipole and loop antenna, respectively.

The fabricated antennas have been characterized using a Rhode \& Schwarz ZVB4 VNA calibrated using a standard TOSM calibration kit. The VNA was used to measure the antennas' unloaded reflection coefficient $\left(S_{11}\right)$ response, and upon-loading, the $S_{21}$, as detailed in the next section. Fig. 10 shows the simulated and measured $S_{11}$ of the fabricated positive and negative sensing loops from Fig. 9(d) and (e). The observed good agreement between simulation and measurement validates the models and indicates that the antennas' sensory response will be in-line with the numerical values.

\section{B. Ice Detection and Monitoring}

The first application of the proposed sensor is to detect the formation of ice in real-time. To evaluate the sensor's performance, the antenna has been connected to a VNA's port with a standard $\lambda / 2$ dipole on the second port, as shown in Fig. 11(a). The printed sensing antenna was placed inside a WKL100 environmental chamber, which enables setting the ambient temperature down to $-50^{\circ} \mathrm{C}$, while measuring the antenna's $S_{11}$ and $S_{21}$ response using a precision test cable, as shown in Fig. 11(b). The measured $S_{21}$ will be indicative of the RSS received from an ice-sensing node.

The loop antenna was placed in the environmental chamber at $-20^{\circ} \mathrm{C}$ with a printed $\mathrm{ABS}$ support layer, at a 


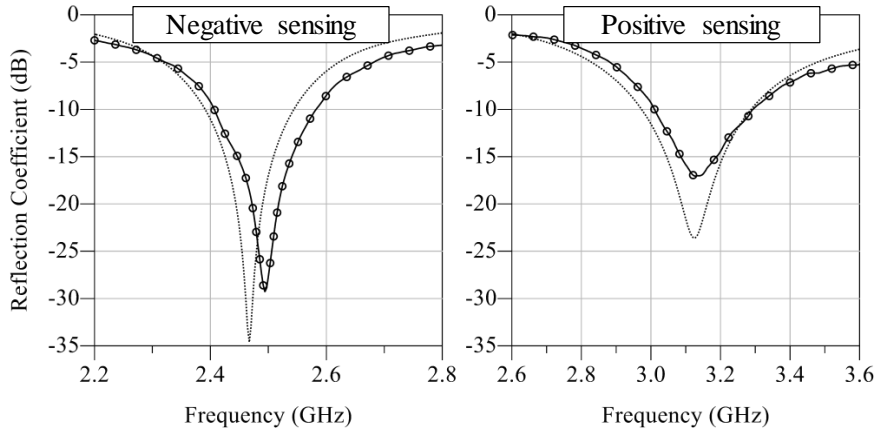

Fig. 10. Simulated (dashed) and measured (solid) reflection coefficient of the loop positive sensing, from Fig. 4(e), and negative sensing, Fig. 4(d), antennas.

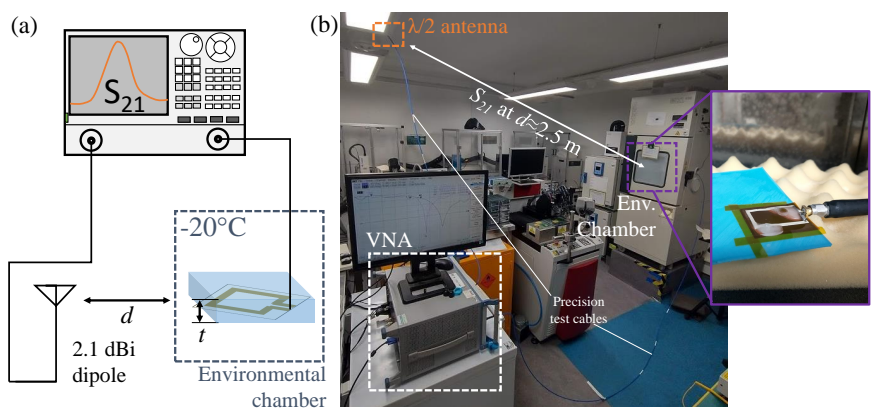

Fig. 11. Measurement setup of the proposed ice sensor: (a) schematic; (b) photograph of the setup utilizing an environmental chamber.

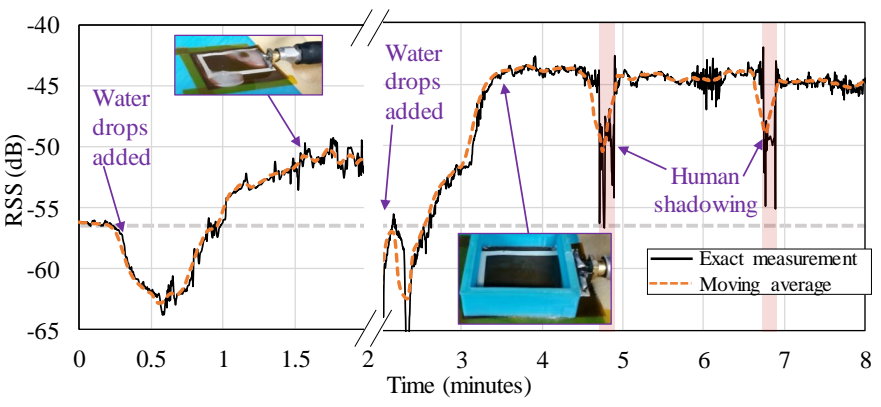

Fig. 12. Measured time-variant RSS $\left(S_{21}\right)$ received from the loop antenna at $2.47 \mathrm{GHz}$ under different loading events; dashed grey line indicates the unloaded bare antenna RSS.

distance $\approx 2.5 \mathrm{~m}$ from the standard dipole. In a real-world application, this distance is limited by the sensitivity of the receiver, which often allows up to $100 \mathrm{~m}$ separation between a typical Bluetooth or Zigbee node and the gateway. The RSS was measured before any loading to be around $-56 \mathrm{~dB}$, as shown at time $=0$ in Fig. 12. Water droplets are then added onto the loop antenna, causing a deterioration in the RSS due to water's high $\tan \delta$. Due to the small volume of water, freezing occurs in 1 minute, which results in an increase in the RSS, beyond the unloaded sensor's response, as shown in minutes 1-2 in Fig. 12. This validates the proposed positive sensing mechanism, where the gain of the antenna has increased by over $4 \mathrm{~dB}$, in response to the small ice formations observed in the inset photograph at minute 2 in Fig. 12.

Further water droplets have been added to the sensor to observe the detection of thicker ice layers. The RSS drop at

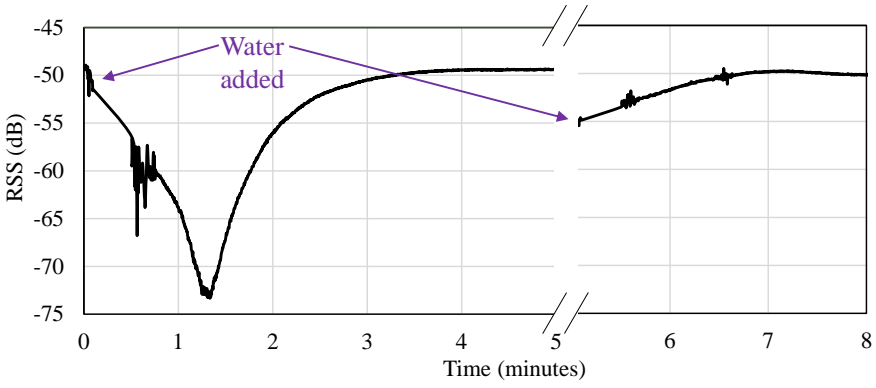

Fig. 13. Measured time-variant RSS from the dipole antenna for two consecutive freeze events.

2-3 minutes is attributed to the losses in the water droplets. As soon as the additional water droplets froze, the RSS is improved by a further $7 \mathrm{~dB}$ compared to the first loaded gain. This indicates that the proposed antenna is not only sensitive to ice forming on its surface but can also be used to measure its thickness.

The same process was repeated for the dipole antenna, from Fig 9(b), to observe its response to freeze events. As observed in Fig. 13, the addition and freezing of water droplets can be detected in real-time, with a similar response to the loop antenna's gain. Nevertheless, by comparing the loaded RSS, at time $=4$ minutes, to the initial RSS, it is observed that the change in the gain is around $1 \mathrm{~dB}$, which is more difficult to detect compared to the loop antenna which exhibited at least $4 \mathrm{~dB}$ sensitivity, which improves to $>10 \mathrm{~dB}$ under thick ice-loading. This is explained by the change in the radiation patterns of the dipole, previously observed in Fig. 8, where the interrogation angle $\approx 40 \circ$ in the broadside direction results in the measurements taking place in the dipole's blind spot. Therefore, it can be concluded that unstable radiation patterns cannot be used to determine absolute gain values. Nevertheless, should the dipole be applied to a real-time sensing system which monitors a water flow, freeze events can be detected through the change in the total efficiency, which results in the gain improvement between 1.2 and 3 minutes in Fig. 13.

From the time-variant RSS measurements, it can be observed that the main sources of uncertainty arise from external factors which would degrade the RSS. For example, in Fig. 9, the line-of-sight (LoS) human body shadowing between the transmitting and the receiving antenna would reduce the instantaneous RSS to a level below the ice detection threshold. However, such LoS blockage is likely to be temporal, and will not influence the performance of the system over a long sampling period, and can be filtered out using a moving average filter. As shown in Fig. 12, the moving average trace is not susceptible to the introduced interference, and will enable a correct detection of the ice.

The presence of water droplets around the antenna, in presence of the ice, can also mask the sensor's response. This is observed in Fig. 12 at $2-3$ minutes, where the measured channel gain degrades when water droplets were added to an iced antenna. This is due to the water acting as an absorptive medium which degrades the antenna's efficiency. However, 


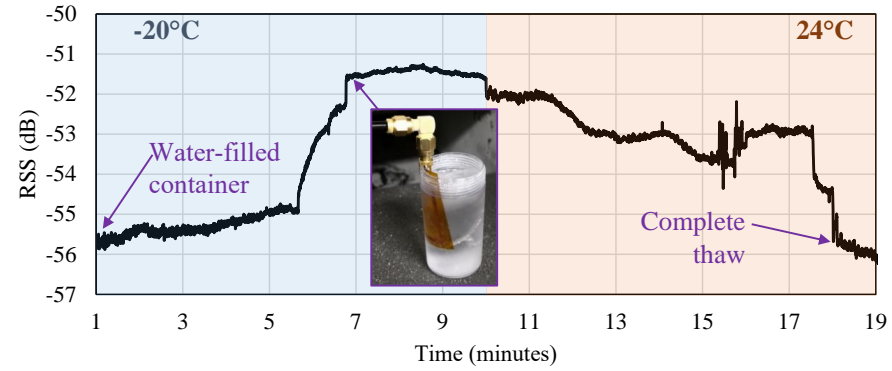

Fig. 14. Measured time-variant RSS $\left(S_{21}\right)$ from the submerged loop antenna emulating an in-pipe IoT monitoring system application.

interference from water will also be temporal, where the ambient freezing temperature in a deployment environment will result in the additional water droplet (from precipitation or dew) freezing, as observed in Fig. 12, from minutes $3-$ 3.5. Therefore, while unavoidable temporal variations in the measured gain can mask the sensor's response, the objective of detecting the presence of ice can still be met over a longer sampling period.

A key application of ice and frost detection is frozen water pipes [5]. As the proposed antenna is implemented on a flexible substrate, it can conform to fit inside water pipes. A plastic tube was used as a water container where the antenna is attached and placed inside the environmental chamber, set to $-20^{\circ} \mathrm{C}$. The tube was filled with water submerging the embedded antenna. The measured time-variant $S_{21}$ of the freezing water pipe is shown in Fig. 14, with the inset showing a photograph of the tube following a complete freeze. The observed RSS change of over $4 \mathrm{~dB}$ to the water freezing demonstrates that the proposed sensor could instantly detect the presence of ice. Given that the $S_{21}$ measurement was performed inside the environmental chamber's enclosure, which combines several metallic and insulating surfaces, it can be concluded that freeze events could be detected using deeply embedded antennas in inaccessible locations, making the proposed sensor highly suitable for embedded sensing applications.

\section{Remote Ice Thickness Measurements}

In addition to the ability of the sensor to detect the presence of a dielectric superstrate, i.e. the ice, it can be used to wirelessly measure its thickness. This was observed through the linear relation between the thickness and the antenna's gain, in Fig. 5, which was chosen as the PUT. A similar setup to Fig. 11(a) was used to remotely measure the ice thickness $t$, with the exception of the measurements taking place outside the environmental chamber, to avoid variations in the antenna's radiation patterns due to the metal enclosure. 3D printed molds were fabricated with different heights to contain the ice used in the thickness investigation. Fig. 15 shows the broadband measured $S_{21}$ /RSS between the sensing antenna and the "reader" dipole, along with the $S_{11}$ of the loop.

The simulated ice thickness-gain relation, from Fig 5(a), has been used to calculate the thickness of the ice, based on the

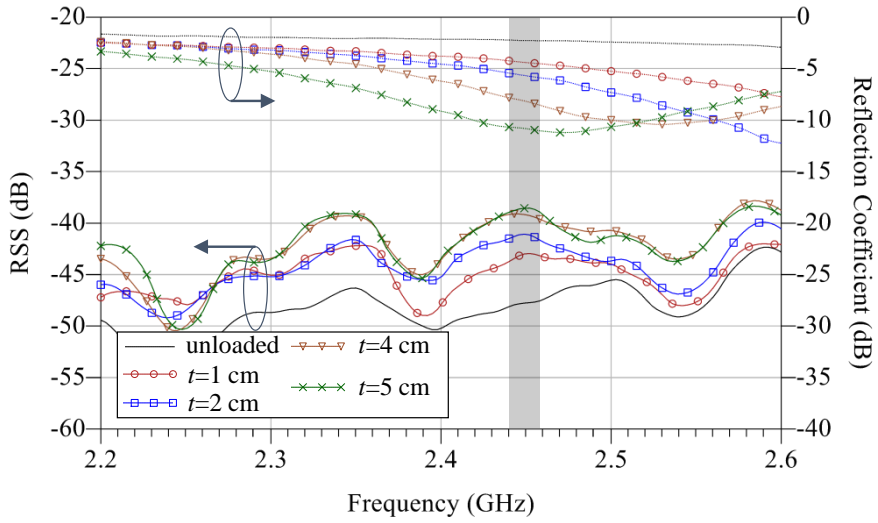

Fig. 15. Measured RSS between the sensing loop and the standard dipole, along with the reflection coefficient of the sensing antenna for varying ice thicknesses.

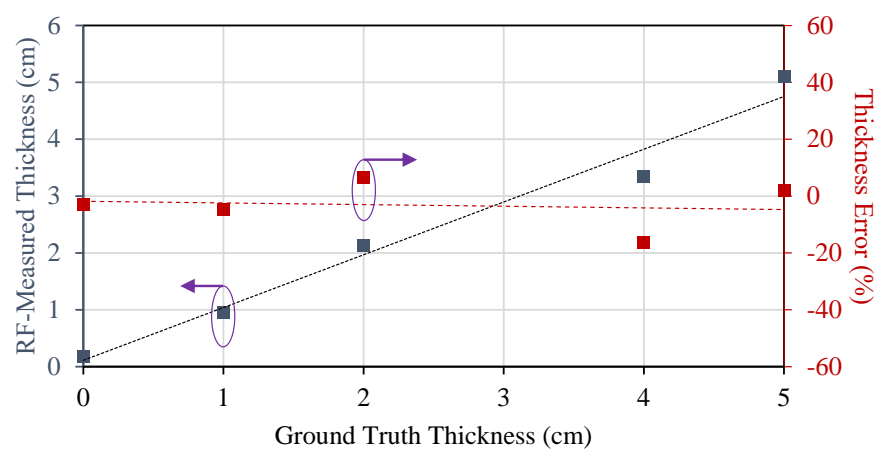

Fig. 16. Remotely measured ice thickness based on the gain and the relative measurement error for $0<t<50 \mathrm{~mm}$.

measured variation in the antenna's gain. The thickness of the ice has been evaluated using

$$
t=z \times \exp \left(\frac{\Delta G-x)}{y}\right),
$$

where $x$ and $y$ are the curve fitting exponents obtained from the CST simulated thickness-gain relation, in Fig. 5(a), $\Delta G$ is the measured change in the RSS, caused by the antenna's gain improvement, and $z$ is an empirical tuning parameter, to account for the measurement tolerances due to air gaps between the antenna and the placed ice samples.

Fig. 16 shows the RF-measured ice thickness compared to the ground truth thickness, where it can be observed that the maximum error percentage obtained with under $20 \%$. Averaging the measurement error across the different ice thicknesses investigated, the sensor achieves a $91 \%$ accuracy in remotely quantifying the thickness of the ice layer over the antenna, using wireless far-field gain measurements. Therefore, the proposed sensor could be reliably used to not only detect frost events, but also to quantify the accumulating ice, enabling its use in wind turbine monitoring [35]. While the $S_{11}$, in Fig 15, was found to exhibit a similar measured response to thicker ice layers, when the $t$ was evaluated based on the $S_{11}$ the average error was found to be around $40 \%$. This further validates the results in Section III, where the far-field $G$ was found to be the most linear PUT. Furthermore, the linear and accuracte response of $G$ as the PUT validates the proposed methodology 
TABLE I

COMPARISON WITH RECENT RF ICE SENSORS

\begin{tabular}{|c|c|c|c|c|c|}
\hline & $\begin{array}{l}\text { This } \\
\text { work }\end{array}$ & $\begin{array}{l}2021 \\
{[20]}\end{array}$ & $\begin{array}{l}2021 \\
{[26]}\end{array}$ & $\begin{array}{l}2021 \\
{[27]}\end{array}$ & $\begin{array}{l}2019 \\
{[21]}\end{array}$ \\
\hline $\begin{array}{l}\text { Sensor } \\
\text { type }\end{array}$ & $\begin{array}{l}\text { Loop } \\
\text { antenna }\end{array}$ & $\begin{array}{l}\text { RFID } \\
\text { tag [39] }\end{array}$ & $\begin{array}{l}\text { Patch } \\
\text { Antenna }\end{array}$ & $\begin{array}{l}\text { Patch } \\
\text { Antenna }\end{array}$ & $\begin{array}{l}\mu \text { strip } \\
\text { Res- } \\
\text { onator }\end{array}$ \\
\hline $\begin{array}{l}\text { Freq. } \\
\text { (GHz) }\end{array}$ & 2.4 & 0.868 & 2.4 & 3.9 & $3.5-5.0$ \\
\hline PUT & $G$ & $G$ & $G$ & $S_{11}$ & $S_{21}$ \\
\hline $\begin{array}{l}\text { Wireless } \\
\text { read }\end{array}$ & Yes & Yes & Yes & No & No \\
\hline $\begin{array}{l}t \quad \text { meas. } \\
\text { range }\end{array}$ & $\begin{array}{l}\mathbf{0 - 5 0} \\
\text { mm }\end{array}$ & $\mathrm{NA}^{*}$ & $\begin{array}{l}0-25 \\
\mathrm{~mm}^{\dagger}\end{array}$ & $8 \mathrm{~mm}$ & NA $^{*}$ \\
\hline $\begin{array}{l}t \\
\text { accuracy }\end{array}$ & $>90 \%$ & $\mathrm{NA}^{*}$ & NA & $\approx 70 \%$ & $\mathrm{NA}^{*}$ \\
\hline $\begin{array}{l}\text { Elec. } \\
\text { size }\left(\lambda^{2}\right)\end{array}$ & $\begin{array}{l}0.28 \times \\
0.15\end{array}$ & $\begin{array}{l}0.32 \times \\
0.01\end{array}$ & $\begin{array}{l}0.48 \times \\
0.54\end{array}$ & $\begin{array}{l}\approx 0.4 \times \\
0.4\end{array}$ & $\begin{array}{l}0.56 \times \\
0.70\end{array}$ \\
\hline
\end{tabular}

${ }^{*}$ No $t$ measurement, detection-only sensor; $†$ Simulated thickness, no experimental thickness measurement; ${ }^{\ddagger}$ evaluated from the graph.

of systematically investigating the different PUTs to reach the optimal RF sensing performance, unlike previous sensors where the PUT choice was not justified with respect to the sensitivity [20], [26], [27].

\section{RF-Enabled Ice Sensing in Future IoT Networks}

The proposed sensor could be applied in a variety of industrial and smart cities IoT usecases, including critical infrastructure monitoring [2] and energy generation [35].A single IoT-connected gateway could act as a sink for the sensor output from several data acquisition nodes, based on the proposed antenna. The conformable sensing antennas could be deeply-embedded within water or gas pipes, or retrofitted as "smart-skin" on important assets or surfaces [2], [36]. A range of low-power single-chip $2.4 \mathrm{GHz}$ transceivers have been proposed [37], which could integrate with the printed antenna for pervasive deployment in a smart city environment. By measuring the sensor nodes" unloaded RSS and then monitoring their time-varying RSS [20], the build-up of ice could be rapidly detected even during temporal LoS shadowing, as previously observed in Fig. 12.

In addition to using an active transmitter for wireless detection, the developed sensor based on the proposed sensing methodology could be integrated in a variety of IoT node architectures. Recalling Fig. 15, both the antenna's far-field gain and 1-port $S_{11}$ could be utilized to detect the presence of ice and measure its thickness. Therefore, the developed loop antenna can be integrated in either of the read-out circuits in Fig. 1(a) and (b). Furthermore, should the antenna be connected to a $50 \Omega$-matched RF energy harvesting rectifier [38], a passive RFID system, as in 1(c). could also utilize the proposed antenna for ice detection.

Table I compares the developed loop sensor to recently reported RF-based ice sensors, where it can be observed that the proposed sensor achieves the highest accuracy and thickness measurement range. Moreover, the proposed solution is the most scalable to different frequency bands, antenna designs, and read-out circuits. For example, the resonators in [21], [24] can only be interrogated using the active circuitry shown in Fig. 1(a), increasing the complexity beyond that of a typical IoT node. Furthermore, while antenna-based farfield sensing at $2.4 \mathrm{GHz}$ was proposed in [26], the patch antenna's gain change is under $1 \mathrm{~dB}$, implying that multitone measurement is required across the $2.4-2.5 \mathrm{GHz}$ spectrum to identify the resonant peak, in addition to requiring more sensitive detector/ADC circuitry to resolve the $<1 \mathrm{~dB}$ gain change. Finally, while battery-free ice sensing RFID tags were proposed in [20], the approach is limited to complex-conjugate RFID antennas and is not transferable to different frequency bands or communication protocols. In addition, the read range of such RF-powered RFID sensors will be restricted by the RFID tag sensitivity, which can only be interrogated up to $10 \mathrm{~m}$ [39].

\section{CONCLUSION}

In this paper, a standard methodology was proposed and investigated for designing RF sensors, towards enabling low-cost and pervasive RF-enabled sensory IoT. Remote ice detection and monitoring was investigated presenting a loop antenna sensor operating at $2.4 \mathrm{GHz}$, with a state-of-the-art sensory response. The key findings can be summarized as

1) The sensors' PUT is the most influential variable which controls the accuracy, where $>90 \%$ accuracy was achieved by interrogating the gain instead of the $S_{11}$;

2) Positive sensing, where the antenna's gain improves in response to lading, in this case ice build-up, presents the highest linearity and immunity to interference;

3) The stability of the radiation patterns of a sensing antenna is paramount, and could render certain antenna designs, e.g. the printed dipole in this study, unsuitable for their wireless sensing application.

Compared to recently reported sensors, the proposed flexible and printed antenna is highly suitable for rapid and accurate wireless detection of ice in future smart cities. It is anticipated that the proposed methodology will underpin various RF-enabled sensing applications, simplifying the design and implementation of RF-enabled IoT wireless sensors.

\section{REFERENCES}

[1] I. Zhou, J. Lipman, M. Abolhasan, N. Shariati, and D. W. Lamb, "Frost monitoring cyber-physical system: A survey on prediction and active protection methods," IEEE Internet of Things Journal, vol. 7, no. 7, pp. $6514-6527,2020$

[2] L. Russell, R. Goubran, F. Kwamena, and F. Knoefel, "Agile iot for critical infrastructure resilience: Cross-modal sensing as part of a situational awareness approach," IEEE Internet of Things Journal, vol. 5, no. 6 , pp. 4454-4465, 2018.

[3] K. Nakata, K. I. Ohshima, and S. Nihashi, "Estimation of thin-ice thickness and discrimination of ice type from amsr-e passive microwave data," IEEE Transactions on Geoscience and Remote Sensing, vol. 57, no. 1, pp. 263-276, 2019.

[4] K. Wei, Y. Yang, H. Zuo, and D. Zhong, "A review on ice detection technology and ice elimination technology for wind turbine," Wind Energy, vol. 23, no. 3, pp. 433-457, 2020

[5] "Frozen pipes cause billions in damage, but can be prevented," Insurance Journal, 2006, Online: $\quad$ https://www.insurancejournal.com/magazines/magfeatures/2006/01/02/64842.htm. [accessed: 12/03/2021.

[6] J.-B. Yan, S. Gogineni, B. Camps-Raga, and J. Brozena, "A dualpolarized 2-18-ghz vivaldi array for airborne radar measurements of snow," IEEE Transactions on Antennas and Propagation, vol. 64, no. 2, pp. 781-785, 2016. 
[7] A. Carta, A. Ghaghazanian, R. Stefanelli, and D. Trinchero, "Radiofrequency sensors for snow conditions monitoring and real time avalanche alerts," in 2013 IEEE Topical Conference on Wireless Sensors and Sensor Networks (WiSNet), 2013, pp. 49-51.

[8] M. A. S. Tajin, C. E. Amanatides, G. Dion, and K. R. Dandekar, "Passive uhf rfid-based knitted wearable compression sensor," IEEE Internet of Things Journal, pp. 1-1, 2021.

[9] B. S. Cook, J. R. Cooper, and M. M. Tentzeris, "An inkjet-printed microfluidic rfid-enabled platform for wireless lab-on-chip applications," IEEE Trans. Microw. Theory Techniq., vol. 61, no. 12, pp. 4714-4723, 2013.

[10] A. A. Abduljabar, D. J. Rowe, A. Porch, and D. A. Barrow, "Novel microwave microfluidic sensor using a microstrip split-ring resonator," IEEE Transactions on Microwave Theory and Techniques, vol. 62, no. 3 pp. 679-688, 2014.

[11] Y. Liu, M. Yu, B. Xia, S. Wang, M. Wang, M. Chen, S. Dai, T. Wang, and T. T. Ye, "E-textile battery-less displacement and strain sensor for human activities tracking," IEEE Internet of Things Journal, pp. 1-1, 2021.

[12] T. Athauda and N. C. Karmakar, "The realization of chipless rfid resonator for multiple physical parameter sensing," IEEE Internet of Things Journal, vol. 6, no. 3, pp. 5387-5396, 2019.

[13] L. Yang, R. Zhang, D. Staiculescu, C. P. Wong, and M. M. Tentzeris, "A Novel Conformal RFID-Enabled Module Utilizing Inkjet-Printed Antennas and Carbon Nanotubes for Gas-Detection Applications," IEEE Antennas Wireless Propag. Lett., vol. 8, pp. 653 - 656, 2009.

[14] Y. He, M. M. Li, G. C. Wan, and M. S. Tong, "A passive and wireless sensor based on rfid antenna for detecting mechanical deformation," IEEE Open Journal of Antennas and Propagation, vol. 1, pp. 426-434, 2020.

[15] X. Wang, J. Zhang, Z. Yu, S. Mao, S. C. G. Periaswamy, and J. Patton, "On remote temperature sensing using commercial uhf rfid tags," IEEE Internet of Things Journal, vol. 6, no. 6, pp. 10715-10727, 2019.

[16] R. Raju, G. E. Bridges, and S. Bhadra, "Wireless passive sensors for food quality monitoring: Improving the safety of food products," IEEE Antennas and Propagation Magazine, vol. 62, no. 5, pp. 76-89, 2020.

[17] E. M. Amin, J. K. Saha, and N. C. Karmakar, "Smart Sensing Materials for Low-Cost Chipless RFID Sensor," IEEE J. Sensors, vol. 14 no. 7, pp. 2198 - 2207, 2014.

[18] R. Ramzan, M. Omar, O. F. Siddiqui, T. S. Ksiksi, and N. Bastaki, "Internet of trees (iotr) implemented by highly dispersive electromagnetic sensors," IEEE Sensors Journal, vol. 21, no. 1, pp. 642-650, 2021.

[19] L. Catarinucci, D. de Donno, L. Mainetti, L. Palano, L. Patrono, M. L. Stefanizzi, and L. Tarricone, "An iot-aware architecture for smart healthcare systems," IEEE Internet of Things Journal, vol. 2, no. 6, pp. 515-526, 2015.

[20] M. Wagih and J. Shi, "Wireless ice detection and monitoring using flexible uhf rfid tags," IEEE Sensors Journal, pp. 1-1, 2021.

[21] B. Wiltshire, K. Mirshahidi, K. Golovin, and M. H. Zarifi, "Robust and sensitive frost and ice detection via planar microwave resonator sensor," Sensors and Actuators B: Chemical, vol. 301, p. 126881, 2019.

[22] P. Jonsson, J. Casselgren, and B. Thörnberg, "Road surface status classification using spectral analysis of nir camera images," IEEE Sensors Journal, vol. 15, no. 3, pp. 1641-1656, 2015.

[23] Y. H. Tai, A. S. A. Kamal, Y. J. Park, P. C. Tsai, Y. L. Ho, P. K. Wei, H. Daiguji, and J. J. Delaunay, "Real-time monitoring of frost/defrost processes using a tapered optical fiber," IEEE Sensors Journal, vol. 21, no. 5, pp. 6188-6194, 2021.

[24] R. Kozak, B. D. Wiltshire, M. A. R. Khandoker, K. Golovin, and M. H. Zarifi, "Modified microwave sensor with a patterned ground heater for detection and prevention of ice accumulation," ACS Applied Materials and Interfaces, vol. 12(49), pp. 55 483-55 492, 2020.

[25] J. Shi and M. Wagih, "Flexible Direct-Write Printed RF Sensor for RF Ice Sensing," in 2021 IEEE International Conference on Flexible and Printable Sensors and Systems (FLEPS), 2021.

[26] "Patch antenna sensor for wireless ice and frost detection," Sci. Rep.

[27] R. U. Tariq, M. Ye, X.-L. Zhao, S.-C. Zhang, Z. Cao, and Y.-N. He, "Microwave sensor for detection of ice accretion on base station antenna radome," IEEE Sensors Journal, pp. 1-1, 2021.

[28] J. Bonior, Z. Hu, T. N. Guo, R. C. Qiu, J. P. Browning, and M. C. Wicks, "Software-defined-radio-based wireless tomography: Experimental demonstration and verification," IEEE Geoscience and Remote Sensing Letters, vol. 12, no. 1, pp. 175-179, 2015.

[29] B.-C. Tseng, L.-C. Liao, L.-K. Wu, and H.-T. Lung, "Analytical solutions for the radiated emission of parallel microstrip traces," IEEE Transactions on Electromagnetic Compatibility, vol. 53, no. 3, pp. 842-845, 2011.
[30] V. Komarov, S. Wang, and J. Tang, Permittivity and Measurements. American Cancer Society, 2005.

[31] J. G. D. Hester and M. M. Tentzeris, "Inkjet-Printed Flexible mm-Wave Van-Atta Reflectarrays: A Solution for Ultralong-Range Dense Multitag and Multisensing Chipless RFID Implementations for IoT Smart Skins,' IEEE Trans. Microw. Theory Techn., vol. 64, 12, pp. $4763-4773,2016$.

[32] DuPont, "DuPont Kapton HN," 2019, Online: https://www.dupont.com/ content/dam/dupont/amer/us/en/products/ei-transformation/documents/ DEC-Kapton-HN-datasheet.pdf.

[33] M. Wagih, A. S. Weddell, and S. Beeby, "Meshed High-Impedance Matching Network-Free Rectenna Optimized for Additive Manufacturing," IEEE Open Journal of Antennas and Propagation, vol. 1, pp. 615 $-626,2020$.

[34] M. Wagih, "Direct-Write Dispenser Printing for Rapid Antenna Prototyping on Thin Flexible Substrates," in 2020 European Conference on Antennas and Propagation (EuCAP), 2020.

[35] P. Blasco, J. Palacios, and S. Schmitz, "Effect of icing roughness on wind turbine power production," Wind Energy, vol. 20, no. 4, pp. 601617, 2017.

[36] S. Dey, R. Bhattacharyya, S. E. Sarma, and N. C. Karmakar, "A novel "smart skin" sensor for chipless rfid-based structural health monitoring applications," IEEE Internet of Things Journal, vol. 8, no. 5, pp. 39553971, 2021.

[37] X. Chen, A. Alghaihab, Y. Shi, D. S. Truesdell, B. H. Calhoun, and D. D. Wentzloff, "A crystal-less ble transmitter with clock recovery from gfskmodulated ble packets," IEEE Journal of Solid-State Circuits, vol. 56, no. 7, pp. 1963-1974, 2021.

[38] M. Wagih, A. S. Weddell, and S. Beeby, "Rectennas for RF Energy Harvesting and Wireless Power Transfer: a Review of Antenna Design [Antenna Applications Corner]," IEEE Antennas Propag. Mag., vol. 62 no. 5 , pp. $95-107,2020$.

[39] M. Wagih, Y. Wei, A. Komolafe, R. Torah, and S. Beeby, "Reliable UHF Long-Range Textile-Integrated RFID Tag Based on a Compact Flexible Antenna Filament," Sensors, vol. 20 (12), p. 3435, 2020.

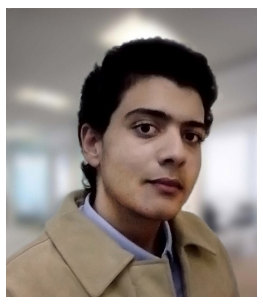

Mahmoud Wagih (GS'18, M'21) received his B.Eng. (Hons.) from the University of Southampton in September 2018, and his Ph.D. in April 2021.

In 2017 he worked as a Research Assistant at the University of Southampton. In 2018, he was a Hardware Engineering Intern at Arm, and, in 2020, a Research Intern at Arm, Cambridge, U.K. He is currently a Research Fellow at the University of Southampton, U.K., leading a Flexible Innovation Fund project on Green RF Sensing. His interests broadly cover antennas and microwave systems in energy harvesting, sensing, and wearable applications. He has 15 journal and 23 conference publications, and has delivered several invited webinars on these topics.

Dr. Wagih is a member of the Institute of Engineering and Technology (MIET). He was the recipient of the Best Undergraduate Project Prize, School Winner Doctoral Award, Best in Faculty Doctoral Research Award, and the Dean's Award, in 2018-2021, at the University of Southampton. He was selected for the IEEE International Microwave Symposium Project Connect in 2019. He received the Best Student Paper Award at the IEEE Wireless Power Transfer Conference, 2019, the Best Oral Paper at PowerMEMS, 2019, and was a Best Student Paper Finalist at IEEE WPTC, 2021. He won the MTT-S Best 3MT Presentation Prize (second place) at the IEEE Microwave Week, 2020, and was a session co-chair at the European Conference on Antennas and Propagation, 2021. He acts as a reviewer for 9 TRANSACTIONS and journals.

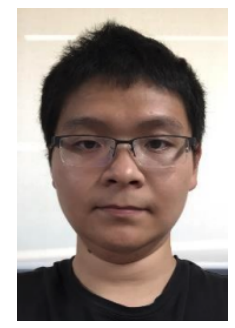

Junjie Shi received a B.Sc. degree from the Nanjing Institute of Technology, China in 2008, an M.Sc. degree in Microelectromechanical systems in 2012, and a Ph.D. in 2017 at the University of Southampton.

He was appointed as a Research Fellow in the School of Electronics and Computer Science (ECS) in 2017. His research interest covers a board range of wearables including but not limited to sensors, actuators, memory, and energy harvesting etc. As well as those wearable devices, he is interested in the fabrication technologies, such as 3D printing, dispenser printing, inkjet printing, micro-fabrications, which enable conventional and novel devices to be directly fabricated on to textiles. 